\title{
A Posteriori Estimates for Cost Functionals of Optimal Control Problems
}

\author{
Alexandra Gaevskaya, ${ }^{1}$ Ronald H.W. Hoppe ${ }^{1,2}$ and Sergey Repin ${ }^{3}$ \\ 1 Institute of Mathematics, Universität Augsburg, D-86159 Augsburg, Germany \\ gaevskaya@math.uni-augsburg. de \\ 2 Department of Mathematics, University of Houston, Houston, \\ TX 77204-3008, USA \\ rohop@math.uh.edu \\ 3 St. Petersburg Division of the Steklov Mathematical Institute, \\ Russian Academy of Sciences, 191011 St. Petersburg, Russia \\ repin@pdmi.ras.ru
}

\section{Introduction}

A posteriori analysis has become an inherent part of numerical mathematics. Methods of a posteriori error estimation for finite element approximations were actively developed in the last two decades (see, e.g., [1, 2, 3, 13] and references therein). For problems in the theory of optimization these methods started receiving attention much later. In particular, for optimal control problems governed by PDE's the literature on this matter is rather scarce. In this work, we present a new approach to a class of optimal control problems associated with elliptic type partial differential equations. In the framework of this approach, we obtain directly computable upper bounds for the cost functionals of the respective optimal control problems.

Let $\Omega \in \mathbb{R}^{n}$ be a Lipschitz domain with boundary $\Gamma:=\partial \Omega$.

Problem 1. Given $\psi \in L_{\infty}(\Omega), y^{d} \in L_{2}(\Omega), u^{d} \in L_{2}(\Omega), f \in L_{2}(\Omega)$, and $a \in \mathbb{R}_{+}$, consider the distributed control problem

$$
\begin{aligned}
& \operatorname{minimize} J(y(v), v):=\frac{1}{2}\left\|y-y^{d}\right\|^{2}+\frac{a}{2}\left\|v-u^{d}\right\|^{2} \\
& \text { over }(y, v) \in H_{0}^{1}(\Omega) \times L^{2}(\Omega), \\
& \text { subject to }-\Delta y=v+f \text { a.e. in } \Omega, \\
& \qquad v \in K:=\left\{v \in L^{2}(\Omega) \mid v \leq \psi \text { a.e. in } \Omega\right\} .
\end{aligned}
$$

The function $y^{d}$ is given and presents the desired shape of the state function $y$, whereas $u^{d}$ presents the desired control. It is well-known that under the above assumptions Problem 1 has a unique solution (see, e.g. [10]). 
There exist many different approaches to optimal control problems of this type. Numerical solution of optimal control problems is usually based on applying specific iterative schemes to the system of optimality conditions, (e.g., the active set strategies or interior point methods; see, e.g., [6],[7] and references therein). Adaptive techniques for optimal control problems governed by PDEs are presented in, e.g., [4] and [9].

In this work, we follow another approach, which is based on so-called functional type a posteriori error estimates. To explain the meaning of such estimates, we consider the Puasson boundary-value problem with homogeneous Dirichlét boundary conditions

$$
\begin{aligned}
-\Delta y=v+f & \text { in } \quad \Omega \\
y=0 & \text { on } \quad \Gamma
\end{aligned}
$$

which describes the dependence between the control and the state in the optimal control problem (1a)-(1c). Let $\tilde{y}$ be any function from the admissible set $Y:=H_{0}^{1}(\Omega)$, which we view as an approximation of the solution of the elliptic problem (2a)-(2b). It was shown (see, e.g., [11] and [12]) that the error of the approximation $\tilde{y}$ satisfies the following estimate:

$$
\|\nabla(y(v)-\tilde{y})\| \leq\|\tau-\nabla \tilde{y}\|+C_{\Omega}\|\operatorname{div} \tau+v+f\| .
$$

Here $C_{\Omega}$ is the constant in the Friedrichs inequality

$$
\|w\| \leq C_{\Omega}\|\nabla w\|, \quad w \in H_{0}^{1}(\Omega)
$$

for the domain $\Omega$ and $\tau$ is an arbitrary function from the functional class $\Sigma:=$ $H_{\text {div }}\left(\Omega, \mathbb{R}^{n}\right)$. Mathematical justifications of the functional type a posteriori estimates and their analysis can be found in the above cited literature. Below, we recall the main properties of such estimates:

- For any approximation $\tilde{y} \in Y$, the right-hand side of (3) gives an upper bound of the error in the natural energy norm of the problem considered ;

- Its value is equal to zero if and only if $\tilde{y}$ coincides with $y(v)$ and $\tau=\nabla y(v)$;

- The estimate is consistent in the sense that its value tends to zero for any sequences $\left\{\tilde{y}_{k}\right\}$ and $\left\{\tau_{k}\right\}$, converging to the exact solution $y$ and its gradient $\nabla y$, respectively;

- The estimate is exact in the sense that there exists a function $\tau$ such that the inequality holds as the equality;

- The estimate does not depend on the mesh parameters and contains only one global constant.

The function $\tau$ in the expression of the error majorant (3) serves as an image of the exact flux $\nabla y(v)$. It is easy to observe, that two terms of the majorant represent the respective errors in the constitutive relation $\tau=\nabla y(v)$ and in the equilibrium equation $\operatorname{div} \tau+v+f=0$.

In this paper, we apply this estimate in order to reformulate the original optimal control problem. As a result, we obtain a directly computable 
and guaranteed majorant for the cost functional. Besides, we prove that the sequences of approximate state and control functions computed by the minimization of the majorant converge to the exact state and control functions.

\section{Majorants for the Cost Functional}

One of the major difficulties in the problem (1a)-(1c) is that the state and control functions must satisfy the equality constraint presented by the boundaryvalue problem for an elliptic PDE.

Let $v \in K$ and $y \in Y$ be two functions related by the differential equation (1b). For this pair the cost functional is as follows:

$$
J(y(v), v):=\frac{1}{2}\left\|y-y^{d}\right\|^{2}+\frac{a}{2}\left\|v-u^{d}\right\|^{2}
$$

Let $\tilde{y} \in Y$ be a certain approximation of $y$, then we can include it in the first term of the cost functional. By the triangle and Friedrichs inequalities we obtain the estimate

$$
J(y(v), v) \leq \frac{1}{2}\left(\left\|\tilde{y}-y^{d}\right\|+C_{\Omega}\|\nabla(y-\tilde{y})\|\right)^{2}+\frac{a}{2}\left\|v-u^{d}\right\|^{2} .
$$

Now, using the error majorant (3) we can estimate the weak norm of the error and substitute it to the estimate of the cost functional (5). By this procedure, we exclude the explicit entry of the exact solution $y$ of $(2 a)-(2 b)$ from our estimate and arrive at the relation

$J(y(v), v) \leq \frac{1}{2}\left(\left\|\tilde{y}-y^{d}\right\|+C_{\Omega}\|\nabla \tilde{y}-\tau\|+C_{\Omega}^{2}\|\operatorname{div} \tau+v+f\|\right)^{2}+\frac{a}{2}\left\|v-u^{d}\right\|^{2}$.

However, from the computational viewpoint it is desirable to reformulate this estimate in such a way that the right-hand side is given by a quadratic functional. For this purpose, we introduce the parameters $\alpha, \beta>0$. Then, we obtain the following upper bound (hereafter called the Majorant):

$$
J(y(v), v) \leq J^{\oplus}(\alpha, \beta ; \tilde{y}, \tau, v), \quad \forall v \in K,
$$

where

$$
\begin{gathered}
J^{\oplus}(\alpha, \beta ; \tilde{y}, \tau, v):=\frac{1+\alpha}{2}\left\|\tilde{y}-y^{d}\right\|^{2}+\frac{(1+\alpha)(1+\beta)}{2 \alpha} C_{\Omega}^{2}\|\tau-\nabla \tilde{y}\|^{2}+ \\
+\frac{(1+\alpha)(1+\beta)}{2 \alpha \beta} C_{\Omega}^{4}\|\operatorname{div} \tau+v+f\|^{2}+\frac{a}{2}\left\|v-u^{d}\right\|^{2}
\end{gathered}
$$

$\tilde{y} \in Y, \tau$ is an arbitrary function in $\Sigma$.

Remark 1. A similar upper estimate can be derived for the optimal control problem with the cost functional 


$$
\boldsymbol{J}(y, v)=\frac{1}{2}\left\|\nabla y-\sigma^{d}\right\|^{2}+\frac{a}{2}\left\|u-u^{d}\right\|^{2},
$$

where the vector-valuer function $\sigma^{d}$ is given and presents the desired gradient of the state function.

Let us look at the Majorant as at the functional that generates a new minimization problem

Problem $1^{*}$. Given $\psi \in L_{\infty}(\Omega), y^{d} \in L_{2}(\Omega), u^{d} \in L_{2}(\Omega), f \in L_{2}(\Omega)$, and $a \in \mathbb{R}_{+}$,

$$
\begin{aligned}
& \operatorname{minimize} J^{\oplus}(\alpha, \beta ; \tilde{y}, \tau, v) \\
& \text { over } v \in K, \tilde{y} \in Y, \tau \in \Sigma, \alpha, \beta \in \mathbb{R}_{+}, \\
& \qquad K:=\left\{v \in L^{2}(\Omega) \mid v \leq \psi \text { a.e. in } \Omega\right\} .
\end{aligned}
$$

We see that in this problem the differential equation (which in (1a)-(1c) defines the respective admissible set) does not appear explicitly. In (8a)-(8b), the functions $\tau, \tilde{y}$ and $v$ act as independent variables. In the next section, we present properties of the Majorant (7) and show that Problems $1^{*}$ and Problem 1 has one and the same exact lower bound attained on the same state and control functions.

Remark 2. It is worth noting, that the Majorant $J^{\oplus}(\alpha, \beta ; \tilde{y}, \tau, v)$ can be used for finding guaranteed upper bounds for the cost functional when the minimization problem is solved by known methods. Indeed, since the functions $\tilde{y}$ and $v$ are arbitrary, we can take them as approximate solutions computed by certain optimization procedure and minimize the Majorant w.r.t. the function $\tau$ and the parameters $\beta$ and $\alpha$. The respective value $J^{\oplus}$ will show the guaranteed upper bound for the value of the cost functional obtained.

\section{Properties of Majorants}

Theorem 1. The exact lower bound of the Majorant (7) coincides with the optimal value of the cost functional of the problem (1a)-(1c), i.e,

$$
\inf _{\substack{\tilde{y} \in Y, \tau \in \Sigma, v \in K, \alpha, \beta \in \mathbb{R}_{+}}} J^{\oplus}(\alpha, \beta ; \tilde{y}, \tau, v)=J(y(u), u) .
$$

The infimum of $J^{\oplus}$ is attained for $v=u, \tilde{y}=y(u), \tau=\nabla y(u)$.

This property means that our transformation of the original problem is mathematically correct in the sense that the new problem is solvable and has the same lower bound as the original one.

Let $\left\{V_{k}\right\}_{k=1}^{\infty},\left\{Y_{k}\right\}_{k=1}^{\infty}$ and $\left\{\Sigma_{k}\right\}_{k=1}^{\infty}$ be sequences of finite-dimensional subspaces that are limit dense in $V:=L^{2}(\Omega), Y$ and $\Sigma$, respectively. A finitedimensional subset for the control functions we define as $K_{k}:=V_{k} \cap K$. It is 
not difficult to show that $K_{k}$ is limit dense in $K$.

Define the sequence of numbers

$$
J_{k}^{\oplus}:=J^{\oplus}\left(\alpha_{k}, \beta_{k} ; \tilde{y}_{k}, \tau_{k}, v_{k}\right)=\inf _{\substack{\tilde{y} \in Y_{k}, \tau \in \Sigma_{k}, v \in K_{k}, \alpha, \beta \in \mathbb{R}_{+}}} J^{\oplus}(\alpha, \beta ; \tilde{y}, \tau, v),
$$

which is obtained by solving the problem on sequences of the selected finitedimensional subspaces.

Theorem 2. If $K_{k}, Y_{k}$ and $\Sigma_{k}$ are limit dense in $K, Y$, and $\Sigma$, respectively, then

(i) $J_{k}^{\oplus} \rightarrow J(y(u), u) \quad$ as $\quad k \rightarrow \infty$;

(ii) the sequence $\left\{y\left(v_{s}\right), v_{s}\right\}$ converges to the exact solution of the control problem $\{y(u), u\}$ in $Y \times K$.

The Theorem shows that a numerical strategy based upon using the Majorant produces sequences of control and state functions, which make the value of the cost functional as close to the value $J(y(u), u)$ as it is required. Moreover, the respective sequences of the control and state functions tend to the desired solution of the original problem.

\section{Practical implementation}

In this section, we briefly discuss practical implementation of the numerical strategy based on the Majorants. A more detailed exposition of numerical results can be found in [8].

\subsection{Discretization of the problem}

In the resultes exposed below, we restrict ourselves to the case when the problem is solved by usual finite element approximations on a simplicial mesh, which is the same for all functions involved. Let $\mathcal{T}_{h}(\Omega)$ denote such a shaperegular simplicial triangulation of $\Omega$. For the state function we use continuous piecewise affine approximations $\tilde{y}_{h} \in Y_{h}$ vanishing on the boundary $\Gamma$ and for the control $v \in K$ we use piecewise constant approximations $v_{h} \in K_{h}$, where $K_{h}$ is chosen such that $K_{h} \in K$. The vector-valued functions $\tau \in \Sigma$ are approximated by piecewise affine functions $\tau_{h} \in \Sigma_{h}$.

\subsection{Minimization algorithm}

To obtain a sharp upper bound of the cost functional we minimize the Majorant $J^{\oplus}\left(\alpha, \beta ; \tilde{y}_{h}, \tau_{h}, v_{h}\right)$ over $\left(\tilde{y}_{h}, \tau_{h}, v_{h}\right) \in Y_{h} \times \Sigma_{h} \times K_{h}$ and $\alpha, \beta \in \mathbb{R}^{+}$. 
Numerical results presented below were obtained using the following algorithm:

Step 1. Initialization. Set $i=0$, define $\alpha^{0}, \beta^{0}, v_{h}^{0}, \tilde{y}_{h}^{0}$.

Step 2. Minimize $J^{\oplus}\left(\alpha^{i}, \beta^{i} ; \tilde{y}_{h}, \tau_{h}, v_{h}\right)$ over $\left(\tilde{y}_{h}, \tau_{h}, v_{h}\right) \in Y_{h} \times \Sigma_{h} \times K_{h}$.

Step 3. Minimize $J^{\oplus}\left(\alpha, \beta ; \tilde{y}_{h}^{i+1}, \tau_{h}^{i+1}, v_{h}^{i+1}\right)$ w.r.t. $\beta, \alpha \in \mathbb{R}_{+}$. Set $i=i+1$.

Steps 2 and 3 are repeated until

$$
\frac{\left|J_{i}^{\oplus}-J_{i-1}^{\oplus}\right|}{J_{i}^{\oplus}}+\frac{\left\|v_{h}^{i}-v_{h}^{i-1}\right\|}{\left\|v_{h}^{i}\right\|}+\frac{\left\|\nabla\left(\tilde{y}_{h}^{i}-\tilde{y}_{h}^{i-1}\right)\right\|}{\left\|\nabla \tilde{y}_{h}^{i}\right\|}>\epsilon
$$

where $\epsilon$ is a given tolerance and $J_{i}^{\oplus}=J^{\oplus}\left(\alpha^{i}, \beta^{i} ; \tilde{y}_{h}^{i}, \tau_{h}^{i}, v_{h}^{i}\right)$.

\section{Numerical experiments}

The method exposed in the previous sections has been numerically tested on a set of various optimal control problems. In all the examples, it was observed that the sequences of computed upper bounds of the cost functionals rapidly converged to the exact lower bound whose value was computed with high accuracy. Also, it was observed that the sequences of the state and control functions converged to the exact ones.

Below, we show these results on the paradigm of a model problem in the square domain $\Omega=(0,1)^{2}$. In this case, $C_{\Omega}=\frac{1}{\sqrt{2} \pi}$.

The efficiency of the approach is measured by 3 quantities. The index

$$
I=J^{\oplus} / J(y, u)
$$

shows the relation between the value of Majorant computed for the control function $v$ and the exact lower bound of the cost functional $J(y, u)$. The quantities

$$
\eta_{y}=\left(\|y-\tilde{y}\|_{H^{1}} /\|y\|_{H^{1}}\right) * 100 \%, \quad \eta_{u}=(\|v-u\| /\|u\|) * 100 \%,
$$

represent the relative errors in the state and control functions, respectively.

\section{Example}

As an example we take the problem from [6] with the following data: $a=0.01, \psi(x, y)=1, f(x, y)=0, u^{d}(x, y)=0$ and

$$
y^{d}(x, y)= \begin{cases}200(x-0.5)^{2}(1-y) y x, & x \leq 0.5, \\ 200(x-0.5)^{2}(1-y) y(x-1), & \text { else. }\end{cases}
$$

The exact solution of this optimal control problem is unknown. Therefore, to analyze the efficiency of the method we compute the so-called "reference 
solution" using a mesh much finer then those used in subsequent tests. For this task, we have used the primal-dual active set strategy (exposed, e.g., in [5]). The reference value of the cost functional in this case is $J(y, u)=9.5838 \cdot 10^{-2}$.

The discrete problem has been solved for various uniform meshes with $N$ nodes. Table 1 shows the relative errors in the state and control functions and the index $I$.

Table 1.

\begin{tabular}{lllc}
\hline$N$ & $\eta_{y}, \%$ & $\eta_{u}, \%$ & $I$ \\
\hline 25 & 67.51 & 54.39 & 1.050 \\
81 & 31.50 & 25.23 & 1.029 \\
289 & 14.59 & 12.07 & 1.014 \\
1089 & 7.55 & 6.49 & 1.007 \\
4225 & 4.67 & 4.18 & 1.003 \\
16641 & 3.65 & 3.39 & 1.002 \\
\hline
\end{tabular}

In Figure 1, we depict values of the Majorant with respect to the minimization time $(N=1089)$. On this picture, the horizontal line shows $J(y(u), u)$ (actual value of the cost functional) whereas the rapidly decaying curve reflects the reduction of the computable upper bound given by the Majorant. The desired tolerance $\epsilon=10^{-4}$ was achieved after $i=16$ iterations. Approximations $\left(\tilde{y}_{h}, v_{h}\right)$ obtained by the algorithm and the reference state and control functions are displayed on Figure 2. 
Fig. 1. Reduction of the upper bound of the cost functional w. r. t. CPU time.

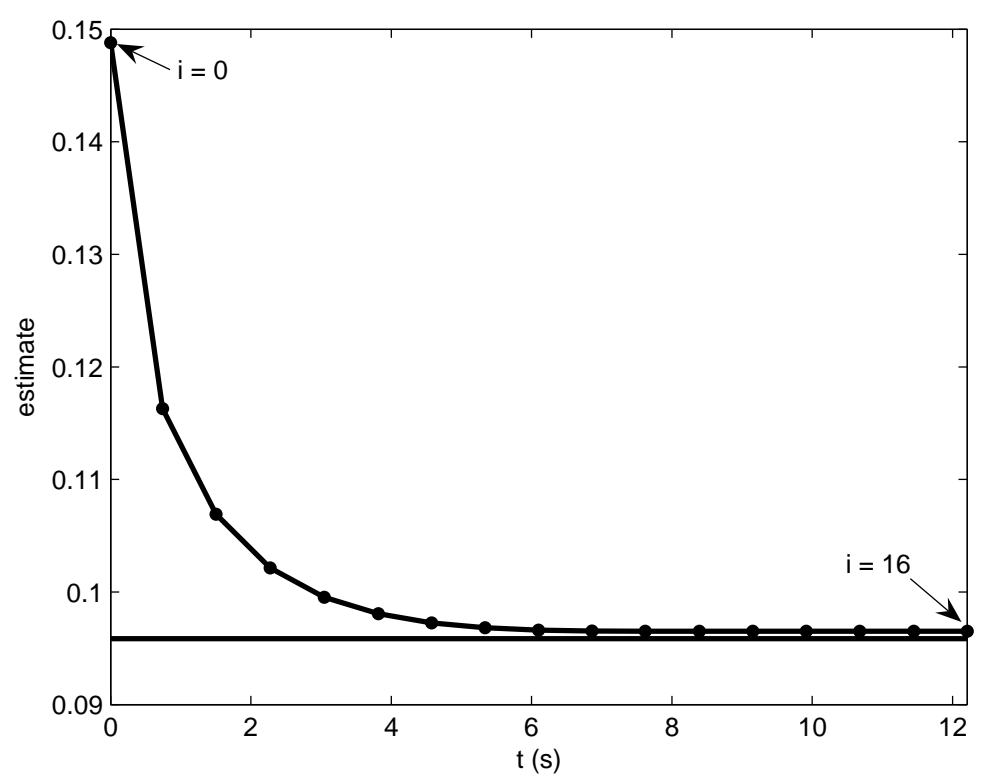

\section{References}

1. M. Ainsworth and J.T. Oden, A posteriori error estimation in finite element analysis. Wiley, New York, 2000.

2. I. Babuška and T. Strouboulis, The finite element method and its reliability, Clarendon Press, Oxford, 2001.

3. W. Bangerth, R. Rannacher. Adaptive finite element methods for differential equations. Birkhäuser, Berlin, 2003.

4. R. Becker, R. Rannacher. An optimal control approach to error estimation and mesh adaptation in finite element methods. In: Acta Numerica (A. Iserles, ed.), 10: 1-102, Cambridge University Press, 2001.

5. M. Bergounioux, M. Haddou, M. Hintermüller, K. Kunisch. A comparison of a Moreau-Yosida-Based Active Set Strategy and Interior Point Methods for Constrained Optimal Control Problems. SIAM J. Optim., Vol. 11, No. 2, pp. 495-521, 2000 .

6. M. Bergounioux, K. Ito and K. Kunisch, Primal-dual strategy for constrained optimal control problems, SIAM J. Control Optim., 37 (1999), pp. 1176-1194.

7. J.F. Bonnans, C. Pola and R. Rebaï, Perturbed path following interior point algorithms, Optim. Methods Softw., 11-12 (1999), pp. 183-210.

8. A. Gaevskaya, R.H.W. Hoppe and S. Repin, Functional majorants for distributed optimal control problems with control constrains (submitted).

9. R. Li, W. Liu, H. Ma, and T. Tang. Adaptive finite element approximation for distributed optimal control problems. SIAM J. Control Optim. Vol. 41, No. 5 (2002), 1321-1349 
Fig. 2. Exact state $y$ (upper left) and approximate state $\tilde{y}_{h}$ (upper right), exact control $u$ (lower left) and approximate control $v_{h}$ (lower right).
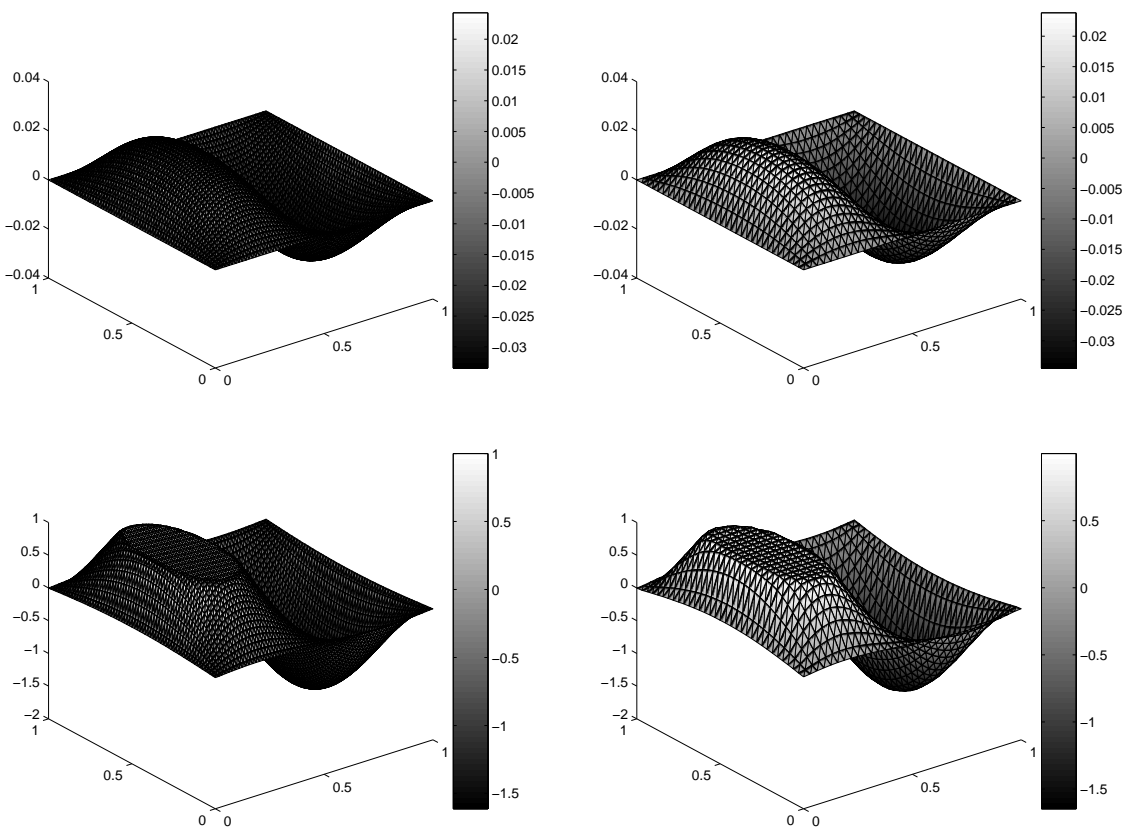

10. J. L. Lions. Optimal Control of Systems Governed by Partial Differential Equations. Springer, Berlin-Heidelberg-New York. 1971.

11. S. Repin, A posteriori error estimation for nonlinear variational problems by duality theory, Zapiski Nauchn. Semin. POMI,243 (1997), 201-214.

12. S. Repin, A posteriori error estimation for variational problems with uniformly convex functionals, Math. Comp., 69 (2000), 230, 481500.

13. R. Verfürth, A review of a posteriori error estimation and adaptive meshrefinement techniques, Wiley and Sons, Teubner, New York, 1996. 